\title{
Comparison of JPEG's competitors for document images
}

\author{
Mostafa Darwiche ${ }^{1}$, The-Anh Pham $^{1}$ and Mathieu Delalandre ${ }^{1}$ \\ ${ }^{1}$ Laboratoire d'Informatique, 64 Avenue Jean Portalis, 37200 Tours, France \\ e-mail: firstname.lastname@univ-tours.fr
}

\begin{abstract}
In this work, we carry out a study on the performance of potential JPEG's competitors when applied to document images. Many novel codecs, such as BPG, Mozjpeg, WebP and JPEG-XR, have been recently introduced in order to substitute the standard JPEG. Nonetheless, there is a lack of performance evaluation of these codecs, especially for a particular category of document images. Therefore, this work makes an attempt to provide a detailed and thorough analysis of the aforementioned JPEG's competitors. To this aim, we first provide a review of the most famous codecs that have been considered as being JPEG replacements. Next, some experiments are performed to study the behavior of these coding schemes. Finally, we extract main remarks and conclusions characterizing the performance of these codecs for different contexts in accordance with OCR performance and PSNR metric.
\end{abstract}

Keywords-Image compression, document encoding, transform coding, coding artifacts.

\section{INTRODUCTION}

With fast increasing 3G-/4G-based markets and infrastructures, online retrieval of document images and exploiting related applications are becoming a crucial need for users with handheld-devices. Customers want to access and retrieve good quality images while expecting a low bandwidth consumption. Additional needs are fast time response and memory-efficient usage. These constraints imply that document images must be encoded and decoded in a very efficient manner. Lossless compression algorithms allow the encoded images to be correctly reconstructed but the gain of compression ratio is not satisfactory. In contrary, lossy coding schemes provide very high compression rate at the cost of losing some certain degree of image quality. Besides, coding quality can be easily controlled by a set of parameters. For these reasons, most multimedia data in practice employ a lossy coding algorithm.

In this work we move out of the box and the standards schemes and run experiments on some of the famous coding schemes $\left(\mathrm{BPG}^{1}, \mathrm{Mozjpeg}^{2}, \mathrm{WebP}^{3}\right.$ and JPEG-XR ${ }^{4}$ ), where all of them are competing with the $\mathrm{JPEG}^{5}$ standard, and might be fair successors. Several attempts were done [17] [18] and showed that these codecs have beaten the standard JPEG for natural images. However, the tests were conducted on small datasets and the results were evaluated by computing different similarity quality metrics (Y-SSIM [2], RGB-SSIM [2], IW-SSIM [8] and PSNR-HVS-M [4]). A relevant work

\footnotetext{
${ }^{1}$ http://bellard.org/bpg/

${ }^{2}$ https://github.com/mozilla/mozjpeg

${ }^{3}$ https://developers.google.com/speed/webp/download

${ }^{4}$ https://jxrlib.codeplex.com/

${ }^{5}$ http://www.ijg.org/
}

in [6] concerns assessing quality of common image formats (e.g., JPEG, TIFF, and PNG) that relies on optical character recognition (OCR) errors and peak signal to noise ratio (PSNR) metric. The authors in [3] compare the performance of different coding methods (JPEG, JPEG 2000, MRC) using traditional PSNR metric applied to several document samples. Since our target is to provide a study on document images, we then use a large dataset with different image resolutions, compress them at very low bit-rate and after that evaluate the output images using OCR accuracy. We also take into account the PSNR measure to serve as an additional quality metric. The main goal of this study is to make a clear statement about performance of JPEG standard and its competitors on document images in terms of compression rate, coding quality, artifact distortion and processing time.

The rest of this paper has been oganized as follows. In Section II, we provide a review of the most potential competitors of the JPEG standard. Next, Section III details the evaluation protocol, experimental settings and evaluation metrics. Section IV presents the detailed results along with an open discussion of all the studied codecs. Finally, Section V concludes the paper and gives several directions of potential future researches.

\section{REVIEW OF JPEG'S COMPETITORS}

\section{A. Better Portable Graphics (BPG)}

BPG [12] is a new lossy image compression technique. It is based on the latest video compression standard High Efficient Video Coding (HEVC [9]) which has been developed by the Joint Collaborative Team on Video Coding (JCTVC). BPG uses a subset of JCTVC (default) and x265 HEVC encoders. Both share the same workflow for encoding/decoding and implement the essential tools of HEVC. However x265 is considered as a fast implementation of HEVC, it offers different encoding settings and reflects a fair trade-off between compression rate and encoding speed. Also, x265 is developed based on single instruction multiple data (SIMD) and multimedia extensions (MMX) technologies [19]. Consequently, it could be much faster than JCTVC but with lower compression quality.

Figure 1 shows the subset of HEVC encoder and decoder used in BPG [10]. Essentially, HEVC is a video compression technique based on a hybrid coding scheme which involves inter/intra predictions, transform and entropy coding after decomposing input pictures into varying size coding tree blocks (CTB). The inter prediction mode explores the spatial 
redundancy between successive frames, while the intra prediction mode exploits the spatial redundancy within a single frame. Consequently, for still picture coding, it is possible to use HEVC with intra prediction mode as BPG does. As shown in Figure 1, the encoder is an iterative process composing of three main blocks: intra prediction, encoding (i.e., transform/quantization), and decoding (i.e., inverse transform/quantization). All of these blocks are processed on the variable size CTB representation. That means the size of CTB can be flexible (i.e., $16 \times 16,32 \times 32,64 \times 64$ ). By using a flexible and adaptive size, HEVC enables the encoder to exploit better spatial redundancy, especially when working with high resolution images.

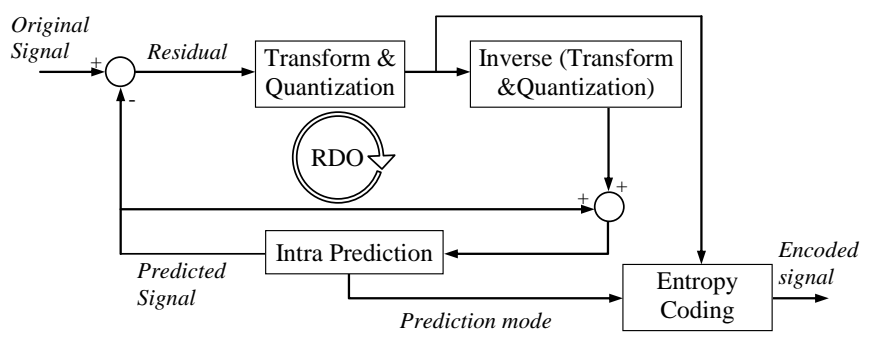

Fig. 1. Subset of HEVC encoder used by BPG.

Each CTB is then divided into coding units (CU), each of which can be further partitioned forming a quad-tree decomposition that minimizes the rate distortion (RD) cost (known as recursive quad-tree splitting process). Every CU consists of a coding block (CB) for luminence and two CBs for chrominance along with syntax elements. Each $\mathrm{CU}$ will be the root of one or multiple prediction units (PU) and transform units (TU). PU and TU have the same components as CU (i.e., one luma block, two chroma blocks and syntax elements). PU is computed based on intra-picture prediction which provides up to 35 prediction modes (33 angular, planar and direct current (DC)) applied to the neighbors of the current $\mathrm{CU}$. Each prediction mode indicates a different direction to compute the block predictor, making it efficiently fitting to the block content characteristics. TU holds the residual which is computed as the difference of the prediction and the original blocks. The size of the square transform block (TB) can be varied from $4 \times 4$ to $32 \times 32$. Except for the smallest TB $(4 \times 4)$ which uses the discrete sine transform (DST), the others are encoded using discrete cosine transform (DCT).

Originally, HEVC performs rate distortion optimization (RDO) by using the Lagrangian encoding technique [11] applied in 3 modes: mode decision, motion estimation and quantization. However for BPG, the motion estimation is ignored. Mode decision emerges primarily in the selection of the intra prediction modes (i.e., the best one out of 35 directions), the selection of transform size of $\mathrm{CU}$, and the decision whether to split a CU into more smaller CUs. The best combination coding mode is selected so as to minimize the RD cost. As for the quantization and for each TB, it computes several flags to indicate whether the TB holds nonzero transform coefficient levels or not, the last nonzero level index, the presence of nonzero levels in the subblocks and some other syntax elements.

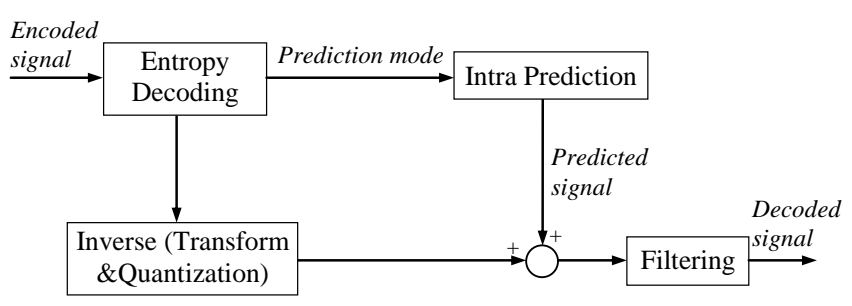

Fig. 2. Subset of HEVC decoder used by BPG.

For the decoding side as shown in Figure 2, the encoded signal is first processed by the entropy decoder to yield the quantized transform coefficients (QTC) of the residuals accompanying with the prediction mode. Next, dequantization is applied to these coefficients followed by inverse transformation to reconstruct the residual in spatial space. The resulting residuals are then added with the predicted signal to form the complete signal which is eventually undergone a filtering process (i.e, deblocking and sample adaptive offset filters) to reduce coding artifacts.

\section{B. Mozjpeg}

Mozjpeg is an open license project established by Mozilla community [13]. Its main target is to provide a web encoding workflow. Applying one instruction on array/vector of data with multiple processors is determinedly faster than running the instruction on each data value (pixel in case of image), this approach is well known and used commonly for image/video encoders. Mozjpeg applies during the compression the Huffman trees and progressive scans, also the Trellis quantization technique which is based on carefully determining the details that could be discarded (reduced the losses during quantization stage).

One of the most important features that is included in the latest release of Mozjpeg library (3.0) is the reduction of the ringing artifacts that appears in black and white JPEG images at low bit rate compression (Figure 3 (a) shows the waveform distorted) [14]. This is addressed by increasing the minimum and maximum of the waveform in the DCT domain for sharp edges before applying the quantization. This approach takes care of the clipping of the overshooting caused by the quantization. It extrapolates the waveform with splines to produce rounded minimum and maximum waves. Consequently, it reduces the sharpness of the edges and corners (Figure 3 (b) depicts the waveform after clipping). Nevertheless, this feature is still limited and only deals with artifacts in black-on-white document images.

\section{WebP}

WebP is a new lossy and lossless compression technique found by Google with its main role to speed up the image 


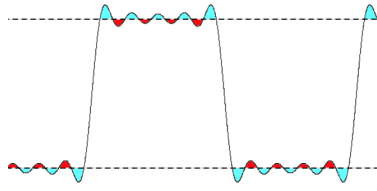

(a)

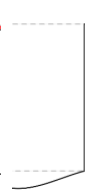

(b)
Fig. 3. Deringing process in Mozjpeg [14]: (a) wzaveform Overshoot (cyan) and undershoot (red); (b) waveform after clipping.

transmission over the network [15]. The lossy compression algorithm of WebP is based on block prediction for image encoding, the same one used in VP8 (video codec found by Google as well). In the same spirit as the BPG codec, the idea is to compute the difference between actual values in a block and the predicted ones based on the neighbor (intra) blocks; this residual will be encoded during the compression. It is worthy to mention that WebP compression also supports variable block sizes to efficiently exploit the spatial redundancy of local content characteristics. The residuals computed based on block prediction will be transformed using large DCT or ADCT (asymmetric DCT). Next, the transformed coefficients are quantized as usual. Due to the use of residuals in the encoding, the quantized image will contain many zerovalued elements which will be encoded efficiently with entropy encoding. At the end WebP file uses the resource interchange file format (RIFF) for image metadata and encoded stream representation. WebP supports transparency in both lossless and lossy compressions, which could be considered an advantage over JPEG and PNG [15].

\section{JPEG-XR}

JPEG-XR is an image compression format developed by Microsoft to compete with the standard JPEG and to provide a high compression ratio with less artifacts. It supports both lossy and lossless compression in addition to the new features of transparency (alpha channel), fixed and floating point color values and progressive decoding levels [5], [7], [16].

Similar to the JPEG codec, JPEG-XR encoder will subsample the image after converting it to an internal colorspace (luma-chroma colorspace), divide it into blocks, then transform them into the frequency domain and finally apply quantization and entropy coding. However it treats each step differently. First, for the transform coding it uses $4 \times 4$ blocks (it might vary depending on the chroma subsampling used) and applies PCT (Photo Core Transform) which is an integer transformation with lifting scheme. The rationale of using PCT is to avoid the losses caused by DCT. Furthermore, it introduces a filtering step called POT (Photo Overlap Transform) before running the PCT on each $4 \times 4$ block where POT is only presented at low bit-rate compression since its role is to reduce the blocking artifacts. Subsequently, it groups the blocks into macroblocks of size 16x16 for direct and alternating currents (DC/AC) computations (DC and $\mathrm{AC}$ are computed after the DCT transformation). Finally, an adaptive entropy coding will code the DC and AC coefficients, using three techniques including adaptive Huffman coding, DC and AC coefficient prediction scheme, and coefficients reordering. Additionally, JPEG-XR breaks the image into rectangular tiles before encoding, thus decompression of a specific area in the image can be done easily by decoding the needed tiles without the need of decoding the whole image.

\section{EXPERIMENTAL SETTINGS}

Comparative methods: Our evaluation will mainly focus on the aforementioned codecs $\left(\mathrm{BPG}^{1}, \mathrm{MozJPEG}^{2}, \mathrm{WebP}^{3}\right.$ and $\mathrm{JPEG} \mathrm{XR}^{4}$ ). In addition, the JPEG standard $^{5}$ will be also included to serve as a baseline method. For BPG we will run the tests using the default encoder JCTVC and then $\mathrm{x} 265$ encoder. It is worthy pointing out that $\mathrm{x} 265$ encoder supports only binary images.

Evaluation metrics: One of the important aspects of document image quality assessment (IQA) concerns the readability of the inspecting documents. Subjective IQA addressed by human perception is prone to error and is too time-consuming. It is, therefore, preferred to using a machine-based readability measure to assess the document quality. To this aim, OCR performance seems to be the most appropriate measure as most core activities in the field of document image analysis (DIA) concern the development of robust reading systems. In fact, this point was mentioned in [1], that an important measure to reflect machine readability is OCR accuracy. In our experiments, the latest tool ABBYY FineReader $12.04^{6}$ is selected to serve as an OCR system. At the same time, we take into consideration the PNSR measure, as it is the most commonly used to evaluate the quality of decompressed images.

Datasets: We apply our tests on the Medical Archive Records (MAR) dataset for OCR recognition from U.S. National Library of Medecine ${ }^{7}$. This dataset consists of 293 real documents, scanned at 300dpi, from different types of biomedical journals and thus could be useful for our purpose of evaluating the coding distortion on real scenarios. Each document contains several zones accompanying the corresponding groundtruth. Hence, These zones are cropped and saved into seperate images, resulting in 296 images in total. These text images will serve to run OCR performance, while PSNR measures are computed directly on the original 293 documents. To stress all the codecs, we also created another dataset which is the low resolution version $(170 \mathrm{dpi})$ of the original text images and documents. Consequently, OCR and PSNR results are done on both high resolution (300 dpi) and low resolution (170 dpi) images/documents. OCR accuracy is traditionally computed as the ratio of the number of properly recognized characters to the total characters in the groundtruth [6]. The final OCR accuracy and PSNR are averaged, according to the bit-rate, from the results of all the images/documents in the datasets.

\footnotetext{
${ }^{6}$ http://finereader.abbyy.com/professional/features/

${ }^{7}$ http://marg.nlm.nih.gov/
} 


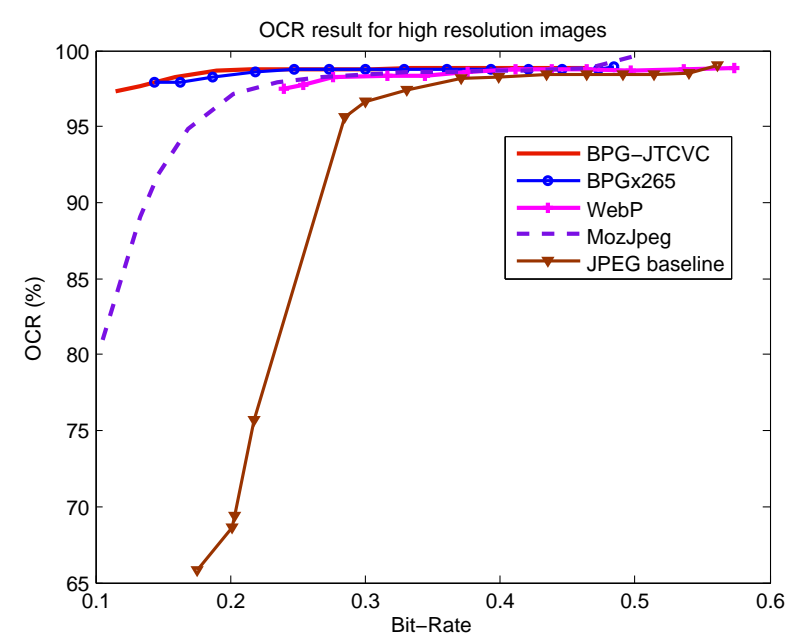

(a)

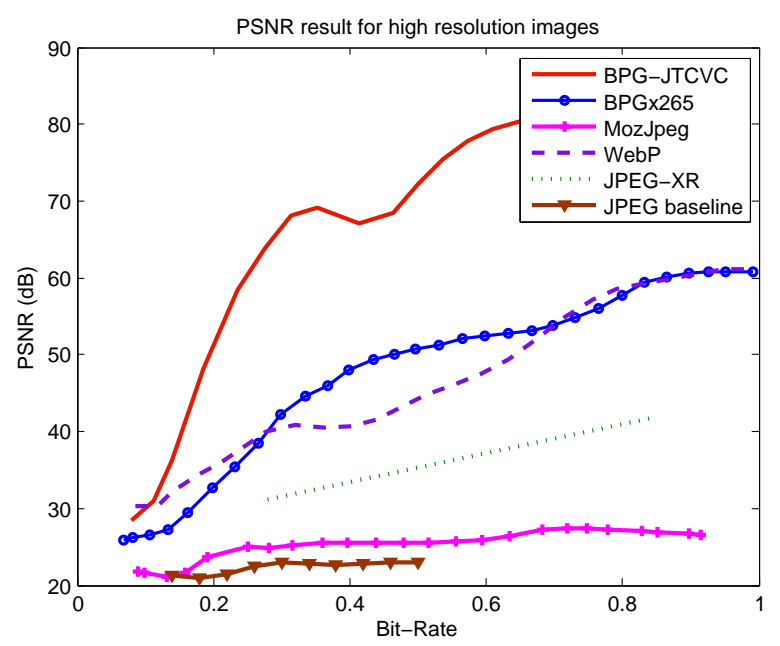

(c)

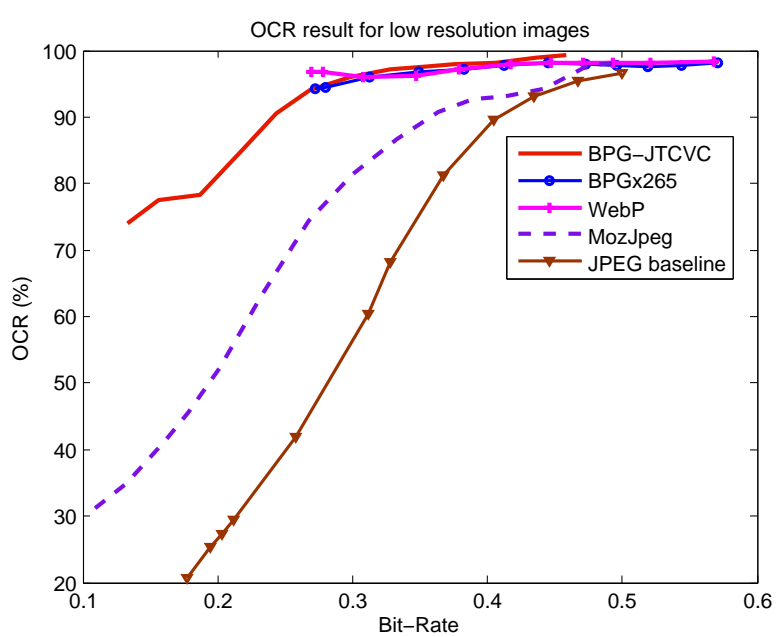

(b)

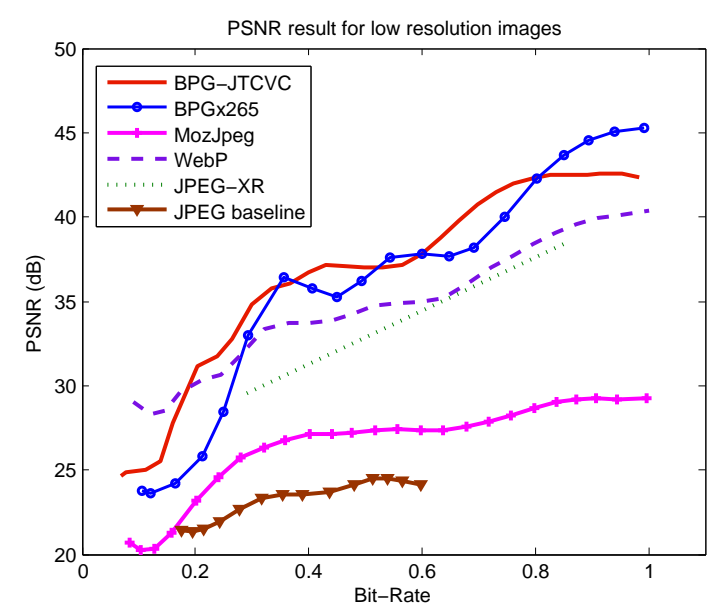

(d)

Fig. 4. OCR accuracy and PSNR results of all the codecs: high resolution (left) and low resolution (right) images.

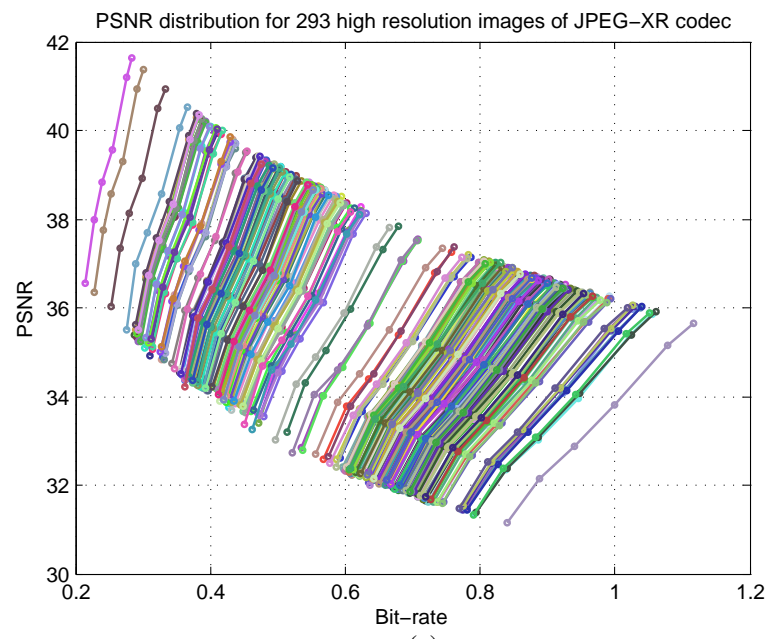

(a)

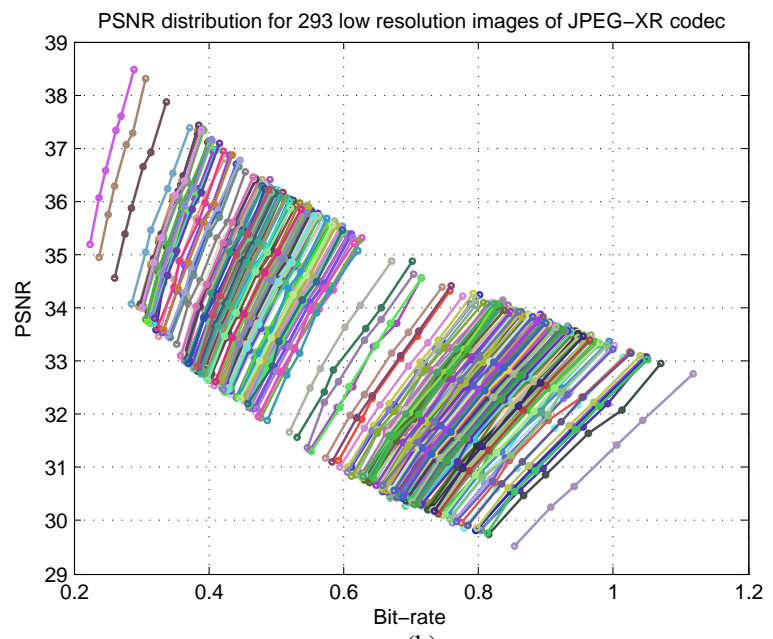

(b)

Fig. 5. PSNR distribution of the JPEG-XR codec: high resolution (a) and low resolution (b) images. Each line refers to an image, where each image is compressed at 6 quality parameters (points on the line) to obtain varying bit-rates/PSNR. 


\section{RESULTS AND DISCUSSION}

Since all the aforementioned codecs (BPG, MozJPEG, WebP and JPEG-XR) are lossy trasform-based coding methods, so they suffer from common artifacts (i.e., blocking and ringing artifcats) at low bit-rate. Blocking artifacts are due to the heavily quantization of the transformed coefficients (i.e., fat distribution of pixel values, high discontinuities between the inter-block boundaries). While ringing artifact refers to the adding of spurious detail along the sharp transitions of the image (i.e., edge locations), this resulted by rough approximation of the high frequency components at low bitrate. In our testing, we would like to highlight the OCR/PSNR behaviors at low bit-rate range (between 0.1 and 0.6) where blocking and ringing artifacts will be introduced, since all the codecs will give close and constant measures (OCR accuracy and PSNR) at higher bit-rates ( $>0.6)$.

\section{A. Evaluation of the codecs based on OCR accuracy}

Figure 4 (a) and (b) illustrate the OCR accuracy behavior at low bit-rates for high and low resolution images, respectively, with text contents for all the codecs except JPEG-XR. The reason why JPEG-XR is excluded, is because it does not provide low bit-rate images $(<0.6)$, even after compressing at the smallest quality parameter $(q=0)$.

1) High resolution images: Based on the graph and at very low bit-rate $(<0.2)$, both BPG's encoders (i.e., JCTVC and $\mathrm{x} 265$ ) perform very efficiently and their performance are very close to each other. We can also see Mozjpeg and WebP have similar actions at that range but both are still a bit less efficient when compared with BPG's encoders. On the other hand, as long as the bit-rate is increasing, the top codecs (i.e., BPG, Mozjpeg and WebP) seem to be very close and provide very high OCR accuracy ( $>96 \%$ ). In contrast, the JPEG baseline is by far outperformed by the other codes at low bit-rates $(<0.3)$, but it starts to give, more or less, the same performance when increasing the bit-rates.

2) Low resolution images: While with the high resolution dataset, all the top codecs (BPG, Mozjpeg and WebP) seem to share quite similar performances, the situation starts to change when working at low resolution images and at low bitrates. The first remark that can be extracted is the significant drop of OCR accuracy for all the codecs when the bit-rate is less than 0.25 (bpp). Also, we can realize that BPG and WebP still keep their same ranks as at high resolution, but Mozjpeg's performance is considerably degraded. As can be seen, Mozjpeg could not provide a good accuracy as BPG's encoders and WebP do. The same for JPEG baseline, where the accuracy is quite low and far from the other codecs.

All these results draw some interesting conclusions. BPG's encoders (JCTVC and x265) are clearly quite robust to compression artifacts in terms of OCR accuracy even for low bit-rates. WebP also works as efficiently as BPG's encoders do, probably because of the similar coding principles between them (i.e., intra prediction, residual encoding, video codec).
However, since WebP does not give lower bit-rates than 0.22 (bpp), it is difficult to judge its real performance at lower bit-rates. For Mozjpeg, because a simple computational preprocessing step was included to reduce the ringing artifacts, it gives excellent results only for high quality images. When this is not the case, it is less effective, yet still much better than the JPEG standard.

\section{B. Evaluation of the codecs based on PSNR}

Figure 4 (c) and (d) show the trade-off between PSNR measures and the bit-rates for high and low resolution images, respectively, of all the codecs. Special care is taken for JPEG$\mathrm{XR}$ in this experiment. Figure 5 shows the PSNR performance for JPEG-XR with high (a) and low (b) resolution datasets for each image (represented by a line). As we can see in Figure 5 (a) and (b), the PSNR distributions for all images in both the datasets show a drop. Consequently, computing the average PNSR values will show the JPEG-XR curve decreasing when moving from left to right (increasing the bitrate). For that reason, JPEG-XR is represented by a linear line drawn between the highest and lowest PSNR values obtained (from Figure 5). This will mainly show, among the others, where JPEG-XR is, to make it comparable with the other codecs (Figure 4 (c) and (d)). In the following, a detailed analysis of all the codecs will be given for both high and low resolution datasets.

1) High resolution images: BPG-JCTVC encoder appears to give an important and excellent coding quality at low bitrates. While BPG with $\mathrm{x} 265$ encoder and WebP look a bit close to each other, but the former is better at the bit-rate greater than 0.3 and the latter is better for the bit-rate less than that. As for JPEG-XR, it is far outperformed by BPG encoders and WebP. On the other hand, JPEG baseline and Mozjpeg apparently behave poorly at low bit-rates where their PSNR values are too low. In contrast to the OCR performance, BPGJCTVC clearly shows its substantial improvement in terms of image quality when compared with all the other codecs.

2) Low resolution images: For low resolutions, basically BPG's encoders and WebP perform in a small PSNR interval, with preference of BPG-JCTVC, BPG-x265 then WebP. Yet, PSNR values are very low for Mozjpeg and the JPEG standard. A good point to mention here is that the gap between the codecs curves is reduced for low resolution images. This is probably due to the lack of details in the images, thus the codecs produce very close output (compressed images).

The difference between BPG-JCTVC and BPG-x265 is that the former is not computationally optimized and takes all the time needed to find the optimized setting having the lowest $\mathrm{RD}$ cost. While the latter is more optimized for faster encoding (it includes different modes/settings). Hence, there is a compromise between the quality (here the PSNR and OCR measures) and the speed of compression. WebP gives superior results comparing to Mozjpeg, JPEG-XR and JPEG baseline, and it is quite close to BPG-x265 encoder. WebP uses the block prediction technique and coding of the signal residuals, 
Table 1. Compression and decompression time (ms) for each codec

\begin{tabular}{|c|c|c|c|c|c|c|c|c|c|c|c|c|c|c|}
\hline \multirow{2}{*}{ Image size } & \multicolumn{2}{|c|}{ BPG-JCTVC } & \multicolumn{2}{|c|}{ BPG-x265 } & \multicolumn{2}{|c|}{ Mozjpeg } & \multicolumn{2}{|c|}{ WebP } & \multicolumn{2}{|c|}{ JPEG-XR } & \multicolumn{2}{|c|}{ JPEG baseline } & \multicolumn{2}{|c|}{ JPEG Turbo } \\
\hline & Com. & Dec. & Com. & Dec. & Com. & Dec. & Com. & Dec. & Com. & Dec. & Com. & Dec. & Com. & Dec. \\
\hline & 41430 & 560 & 4510 & 650 & 2580 & 320 & 750 & 430 & 200 & 200 & 330 & 470 & 130 & 270 \\
\hline $2544 \times 3340$ & 95950 & 910 & 11600 & 1480 & 5380 & 250 & 1550 & 550 & 450 & 350 & 910 & 1260 & 320 & 270 \\
\hline
\end{tabular}

which is similar to the BPG technique (based on HEVC). For that reason, WebP gives quite interesting results.

Mozjpeg results are expected with OCR accuracy measure where we see it competing with BPG's encoders and WebP. However, in terms of PSNR measures, it is far away from the other codecs and close to JPEG baseline. The reason behind this is that Mozjpeg is an improvement of JPEG baseline which include an effective way to deal with compression artifacts by re-shaping the signal amplitudes. So OCR accuracy is good since the images used are only with text contents. But when it comes to PSNR and since the full images might have complicated content (i.e., pictures, graphics, etc.), Mozjpeg could not compress them with the same efficiency. As for JPEG-XR, it is able to serve its main goal which is to beat the JPEG baseline at low bit rate.

\section{Evaluation of running time}

Table 1 provides a report on processing time for all the codecs for different image sizes. These experiments are conducted on the following machine configuration: Windows 7 (64-bit), Intel Core i5 (2.4 GHz), 4Gb RAM. Having a look at the results, BPG compression seems to be very time consuming, especially with JCTVC encoder, even though it gives outstanding performance in terms of both PSNR and OCR results. However, WebP's processing time looks very convenient among the others and also obtained good results when compressing at low bit-rates. In the mean time, JPEG$\mathrm{XR}$ appears to be the fastest competitor codec at compressing and decompressing (due to the use of POT which based on integer transformation), even though the quality metrics (PSNR and OCR) were not good enough compared to BPG and WebP. JPEG Turbo's (accelerated JPEG baseline by using SIMD instructions) processing time is also computed and shows that it is two times faster than JPEG baseline, both gives the same quality measures since they share the same structure and technique compression/decompression.

\section{CONCLUSIONS}

This work aims at providing a study on the performance of JPEG's competitors (BPG, Mozjpeg, WebP and JPEG-XR) for document images. For this aim, we have provided a short review for every codec, and highlighted the techniques used. Extensive experiments have been carried out to characterize the behaviors of the state-of-the-art coding schemes in terms of distortion rate, artifact handling and computational complexity. Future work shall be conducted in terms of developing optimal reconstruction for JPEG encoded images particularly designated to document content.

\section{ACKNOWLEDGMENT}

This work has been supported by the DOD project cofunded by La BPI and Yooz.

\section{REFERENCES}

[1] Ye P., Doermann. Document Image Quality Assessment: A Brief Survey. In 12th International Conference on Document Analysis and Recognition (ICDAR), proceedings, 2013, pp. 723-727.

[2] Z. Wang, A. C. Bovik, H. R. Sheikh and E. P. Simoncelli. Image quality assessment: From error visibility to structural similarity. IEEE Trans. on Image Processing, 13(4): 600-612, 2004.

[3] Mauro Barni, Document and Image Compression, CRC Press, 2006.

[4] N. Ponomarenko, F. Silvestri, K. Egiazarian, M. Carli, J. Astola, V. Lukin. On between-coefficient contrast masking of DCT basis functions, CDROM. In Third International Workshop on Video Processing and Quality Metrics for Consumer Electronics VPQM-07, proceedings, Scottsdale, Arizona, USA, 2007, pp. 4.

[5] Bill, Crow (1 November 2006). "Introducing". Microsoft Developer Network blogs, Bill Crow's blog. Microsoft Corporation. Retrieved 24 October 2009.

[6] G. F. Silva, R. D. Lins. Assessing the OCR degradation in the generation of jpeg, png, and tiff files from adobe pdf. In International Telecommunications Symposium, proceedings, 2010, pp. 1-4.

[7] ISO/IEC 29199-2:2010: Information technology - JPEG XR image coding system - Part 2: Image coding specification

[8] Z. Wang, Q. Li. Information Content Weighting for Perceptual Image Quality Assessment. IEEE Trans. on Image Processing, 20(5): 11851198, 2011.

[9] B. Bross, W.-J. Han, J.-R. Ohm, G. J. Sullivan, and T. Wiegand, High Efficiency Video Coding (HEVC) Text Specification Draft 9, Joint Collaborative Team on Video Coding (JCT-VC) of ITU-T SG 16 WP 3 and ISO/IEC JTC 1/SC 29/WG 11, document JCTVC-K1003, Shanghai, China, Oct. 2012.

[10] J. Vanne, M. Viitanen, T. D. Hamalainen, A. Hallapuro. Comparative Rate-Distortion-Complexity Analysis of HEVC and AVC Video Codecs. IEEE Trans. on Circuits and Systems for Video Technology, 22(12): 18851898, 2012.

[11] J. Ohm, G. J. Sullivan, H. Schwarz, T. K. Tan, T. Wiegand. Comparison of the Coding Efficiency of Video Coding Standards-Including High Efficiency Video Coding (HEVC). IEEE Trans. on Circuits and Systems for Video Technology, 22(12): 1669-1684, 2012.

[12] F. Bellard. BPG Image Format website [Online]. Available: http:// bellard. org/bpg/.

[13] Mozilla. Mozilla Mozjpeg [Online]. Available: https://github. $\mathrm{com} / \mathrm{mozilla/mozjpeg.}$

[14] K. Lesinski. Mozjpeg 3.0 [Online]. Available: http://calendar. perfplanet.com $/ 2014 / \mathrm{mozjpeg}-3-0 /$.

[15] Google Developers WebP. WebP image format - Google Developers [Online]. Available: https://developers.google.com/ speed/webp/ ? csw=1.

[16] JPEG XR Codec Overview. JPEG XR Codec - Windows Dev Center [Online]. Available: https://msdn.microsoft.com/en-us/ library/windows/desktop/hh707223 (v=vs.85). aspx.

[17] Lossy Compressed Image Formats Study. Mozilla Corporation study October 2013 [Online]. Available: http://people.mozilla.org/ josh/lossy_compressed_image_study_october_2013/

[18] Lossy Compressed Image Formats Study. Mozilla Corporation study - July 2014 [Online]. Available: http://people.mozilla.org/ josh/lossy_compressed_image_study_july_2014/

[19] Q. Hu, X. Zhang, Z. Gao, J. Sun. Analysis and optimization of x265 encoder. In Visual Communications and Image Processing Conference, 2014 IEEE, proceedings, Valletta-Malta, December 2014. 